\title{
La didactique de la langue des signes française :
}

Naissance ou reconnaissance d'une discipline à part entière?

The didactics of French sign language: The rise or recognition of a fully-fledged discipline in its own right?

\section{Véronique Geffroy et Élise Leroy}

\section{(2) OpenEdition}

Journals

\section{Édition électronique}

URL : http://journals.openedition.org/tipa/2653

DOI : 10.4000/tipa.2653

ISSN : 2264-7082

\section{Éditeur}

Laboratoire Parole et Langage

Référence électronique

Véronique Geffroy et Élise Leroy, « La didactique de la langue des signes française : », TIPA. Travaux interdisciplinaires sur la parole et le langage [En ligne], 34 | 2018, mis en ligne le 06 septembre 2018, consulté le 09 octobre 2020. URL : http://journals.openedition.org/tipa/2653 ; DOI : https://doi.org/ 10.4000/tipa.2653

Ce document a été généré automatiquement le 9 octobre 2020.

\section{(c)}

La revue TIPA. Travaux interdisciplinaires sur la parole et le langage est mise à disposition selon les termes de la licence Creative Commons Attribution - Pas d'Utilisation Commerciale - Pas de Modification 4.0 International. 


\title{
La didactique de la langue des signes française :
}

\author{
Naissance ou reconnaissance d'une discipline à part entière? \\ The didactics of French sign language: The rise or recognition of a fully-fledged \\ discipline in its own right?
}

Véronique Geffroy et Élise Leroy

\section{Préambule}

1 La surdité perturbe la relation entre des êtres a priori communicants, tous ceux qui entendent sont alors aussi handicapés pour communiquer avec les sourds que les sourds le sont avec ceux qui entendent. Cela a pour incidence d'en faire un handicap partagé et même d'inverser parfois les places : une personne sourde est en quelque sorte surentraînée à pallier les malentendus et elle adopte des stratégies qui facilitent l'intercompréhension, alors que les entendants sont plutôt démunis?. Kahne (2013) l'a bien compris en créant des formations dédiées aux managers d'Airbus, puisque pour lui, la réalité est que les sourds signeurs communiquent plus rapidement et avec plus de précision que les entendants.

2 Par ailleurs, dans toute situation d'apprentissage, il est impératif de « ne pas accepter de ne pas comprendre, ne pas accepter de ne pas se faire comprendre " (Baruk, 1992, in Terrail, 2009, p. 276). Cette position doit même être posée comme une «"exigence éthique" essentielle du métier d'élève comme de la déontologie enseignante » (Baruk, 1992, in Terrail, 2009, p. 276). Cette exigence prend un éclairage bien particulier dans une situation d'enseignement où le professeur doit se faire comprendre d'un ou plusieurs élèves sourds qui doivent aussi, eux, être compris par lui ${ }^{8}$, l'usage d'une langue riche, précoce et confortable dans l'éducation des sourds leur ayant longtemps été refusé.

3 L'histoire de la Langue des Signes Française (LSF) et des particularités socio-culturelles sourdes ${ }^{9}$ a été marquée par des polémiques tenaces. Le fait le plus marquant fut le refus 
généralisé, intervenu en 1880, d'utiliser une langue visuo-gestuelle dans l'instruction des élèves sourds. À peine quelques années plus tard, avec les lois de Jules Ferry, la France adoptera, tout en instaurant la seule langue nationale comme langue d'enseignement, une loi pour scolariser tous les enfants de la République, sauf les sourds et les aveugles dont le sort devait être décidé ultérieurement. À partir de moment-là, pour les enfants sourds "l'enseignement de la parole et la formation professionnelle précéd[èr]ent l'inculcation des connaissances » (Buton, 2009: 311), ce qui a détourné la finalité de l'école pendant longtemps pour eux. La dérive provoquée alors tient au fait que la technicité exigée par la production vocale et la lecture sur les lèvres va "accaparer " toute l'attention pouvant être portée aux contenus scolaires ainsi qu'aux interactions entre professeur et élève(s) sourd(s).

4 Le destin si particulier de la LSF a pu croiser celui d'autres langues d'enseignement possibles, comme les langues régionales ou minoritaires, puisque l'usage de toute autre langue que le français comme langue de scolarisation était devenu impensable pour enseigner jusqu'à la fin du XX ${ }^{\mathrm{e}}$ siècle. Pour autant, même si au début du $\mathrm{XXI}^{\mathrm{e}}$ siècle la reconnaissance de la LSF comme une des langues de France ${ }^{10}$ constitue une avancée remarquable, les études menées dans le champ de la surdité ne se sont pas vraiment attachées à étudier la politique linguistique adoptée envers toutes les langues de la République.

5 Pour ce qui est des questions d'éducation, les méthodes et, par voie de conséquence, la formation des maitres se sont focalisées pendant plus d'un siècle sur la vocalisation, du fait que «la méthode orale implique théoriquement des professeurs qu'ils aient des notions relatives aux mécanismes physiologiques de l'articulation. » (Buton, 2009 : 311). Les recherches concernant les sourds sont tournées prioritairement sur l'acquisition d'un niveau de compétence en français satisfaisant pour l'école, mais analyse-t-on les mêmes « objets " selon que l'on mène des recherches sur la déficience auditive et les actions correctives ou des recherches sur les sourds et la LSF?

Alors qu'un enseignant pourrait s'appuyer sur une communication sans entrave auprès de ses élèves sourds avec une langue visuo-gestuelle de qualité, la modalité des échanges n'étant pas affectée par la déficience auditive, l'utilisation de la LSF en milieu scolaire n'avait survécu, jusqu'au tournant du $21^{\mathrm{e}}$ siècle, que pour les élèves avec handicap associé pour lesquels on avait renoncé à toute ambition. Il faudra attendre le milieu des années 1970 , avec le "réveil sourd ${ }^{11}$ " pour que la question de la langue d'enseignement dans l'éducation des enfants sourds soit ravivée.

7 Assez vite, une association comme « 2 Langues Pour une Éducation » (2LPE) créée fin 1979, au sein de laquelle se retrouvaient des professionnels sourds, des professionnels entendants et des parents d'enfants sourds, avait dénoncé l'échec scolaire avéré des enfants sourds et combattu le choix exclusif du français oral plutôt que celui de la LSF. À peu près au même moment, la perspective d'un nouvel oralisme ressurgissait sur le devant de la scène avec des méthodes ou des outils nouveaux ${ }^{12}$, si bien que la place de la langue visuo-gestuelle dans l'enseignement pour les sourds souleva des questions inédites dans un champ devenu plus polémique encore.

8 Toujours est-il que le point de vue inverse de celui qui s'était imposé depuis un siècle s'est fait entendre: une authentique relation pédagogique peut s'établir entre des individus « autrement parlants » qui cherchent à se faire comprendre et à comprendre en utilisant une langue gestuelle. 
Dans les pages qui vont suivre, nous nous proposons de réfléchir à la branche sur laquelle poser l'étude de la LSF et celle des aspects didactiques qui l'accompagnent, en commençant par regarder comment cette langue est devenue objet d'étude à l'université malgré les résistances.

\section{L'arrivée de la langue des signes à l'université}

\section{Quelques repères historiques}

Dans les années 1960-1970, des linguistes et sociologues américains comme Stokoe et Markowicz (linguistes entendants parmi d'autres au sein du laboratoire de Gallaudet College, Washington DC, États-Unis) avaient commencé à étudier la langue visuogestuelle des sourds - ce qui constituait une nouveauté - à peu près au moment où la sociologie devenait «interactionniste" et où le socioconstructivisme entrait sur la scène scolaire, rappelant dans l'éducation des sourds l'enseignement mutuel qui avait existé avec le « langage mimique », longtemps auparavant.

11 Dans leur sillage, un chercheur français va s'intéresser aux sourds au milieu des années 1970: Mottez, sociologue formé par Touraine au Centre des Mouvements sociaux, bientôt suivi par Cuxac (linguiste) en France ou Grosjean (psycholinguiste) en Suisse.

Les gestes ayant connu plus d'un siècle de déni, ils étaient alors perçus comme un code limité qui ne pouvait pas être pris en compte en tant que langue. De ce fait, les questions autour de l'éducation des sourds étaient résolument ramenées à l'amélioration du français oral, en particulier dans sa modalité articulatoire, ce qui a entretenu une confusion latente que Mottez formulait ainsi, en 1979 : « la surdité étant une pathologie, leur langue en serait une aussi » (in Benvenuto, 2006, p. 252).

Le dynamisme des associations telles, entre autres, IVT ${ }^{13}, \mathrm{ALSF}^{14}$, SERAC ${ }^{15}$ pour Paris et 2LPE au niveau national, ainsi que l'ouverture d'esprit de quelques professionnels dans les institutions spécialisées chargées de l'éducation des sourds, qui ont agi dans leur classe d'abord avec « la porte fermée », produisirent cependant quelques effets (Mato, 2017). Des praticiens entendants tentaient d'analyser leurs pratiques ou menaient des recherches-actions. Parmi eux, ils étaient de plus en plus nombreux à travailler avec des sourds signeurs à leurs côtés au quotidien et quelques-uns n'hésitaient pas à se lancer dans des recherches à caractère scientifique. Dans les années 1980-1990, aux côtés des chercheurs, les acteurs de terrain venaient à l'université pour prendre du recul sur leurs pratiques ou pour les asseoir sur des principes théoriques différents. Ceci explique pourquoi le public des premières manifestations à caractère scientifique était majoritairement constitué de salariés, ayant parfois aussi le statut d'étudiant ou d'auditeur libre.

Toutefois, les sourds, ayant tout à reconstruire après un siècle d'interdiction de leur langue, ne pouvaient prétendre accéder au niveau universitaire et se souciaient donc encore moins d'engendrer un nouveau champ de recherche. Rien sans doute n'aurait pu avancer sans que les quelques universitaires en place offrent un appui inespéré aux sourds signeurs et à un public plus large intéressé par les mêmes thématiques (Mato, 2017). Comme pour tous les chercheurs impliqués dans un paysage polémique, sans nécessairement être des militants engagés, leurs travaux ont contribué à formaliser ce 
qui existait ici ou là et ont permis, par effet cumulatif, la structuration de savoirs jusque-là empiriques.

Même si Mottez, en étant un rouage fondamental des recherches françaises sur les sourds et leur langue, a apporté un étayage au "réveil sourd ", ce sont des travaux en linguistique qui seront les premiers leviers pour une autre éducation des sourds: le bilinguisme. "Les soutenances de thèse de Cuxac et de Bouvet en 1980 et 1981 se démarquent pour avoir été les premières en France à accueillir des interprètes en langue des signes et un auditoire constitué pour une grande part de personnes sourdes.» (Dalle-Nazebi, 2006:420) Il faut citer également le travail de Grosjean, psycholinguiste et professeur honoraire de l'Université de Neuchâtel, qui connut un écho important en France.

Dans un premier temps, la langue visuo-gestuelle des sourds s'introduira au sein des universités françaises comme un sujet assez " exotique », et finalement de plus en plus convoité. Cet objet d'études si singulier, tant pour la recherche où il stimule la réflexion épistémologique que pour les applications pratiques qui en découlent dans l'enseignement, s'implantera d'abord dans le champ de la linguistique, comme une langue à part entière vingt ans avant sa reconnaissance officielle ${ }^{16}$, et permettra finalement d'insinuer la question des sourds dans plusieurs autres champs universitaires.

Les sciences sociales en général s'intéressant aux activités humaines, aux conditions (naturelles et culturelles) et aux résultats de ces activités, les outils des historiens ${ }^{17}$, sociologues $^{18}$, psychologues ${ }^{19}$, philosophes ${ }^{20}$, anthropologues et ethnologues ${ }^{21}$ contribueront vraiment à poser un regard attentif et nouveau sur les usagers de la LSF, pendant une trentaine d'années. Cela dit, la situation complexe et inédite des sourds et de leur langue en France a été difficile à décrire. Un tel objet d'étude ne se laisse pas facilement attraper, abordé tantôt avec un regard similaire à celui porté sur les minorités, tantôt sous l'angle de l'handicapologie.

Ainsi, les années 1980 ont catalysé les prémices de l'entrée de la langue des signes à l'université en France. C'est seulement au cours de la seconde moitié des années 1990 que vont s'ouvrir, à des enseignants-chercheurs entendants travaillant sur la langue des signes, les premiers postes universitaires de maittre de conférence: en sociolinguistique à Rouen en 1994, en linguistique à Paris 8 en 1998, etc. (Garcia \& Burgat, 2016). Ce sera une période au cours de laquelle chercheurs et acteurs du terrain, sourds ou entendants (associations, enseignants, éducateurs, comédiens...) uniront leur force, mus par les mêmes objectifs : diffuser et faire reconnaître cette langue baptisée dorénavant $\operatorname{LSF}^{22}$ comme une des langues de France ${ }^{23}$, offrir une éducation bilingue (LSF- français écrit) aux enfants sourds et donner aux sourds adultes accès aux savoirs et à l'information.

\section{À la recherche d'un ancrage théorique adéquat}

La surdité actualise des facettes mal connues de la capacité humaine à construire du sens $^{24}$. On peut dire que les premiers travaux universitaires sur la LSF ont créé, autour de la communauté sourde, un champ d'études transdisciplinaires, ce qui est assez rare pour être signalé. Quelques linguistes, sociolinguistes, psycholinguistes, (pour ne citer que les disciplines qui sont le plus souvent mobilisées) ont accompagné ensemble une réflexion de fond sur l'utilisation de cette langue dans l'enseignement. Ils se sont 
engagés à titre individuel et ont pu être marginalisés dans un premier temps, mais ils ont contribué à leur échelle à ce que la LSF soit reconnue comme "langue à part entière " en France. Peu nombreuses dans un premier temps, les études se faisaient l'écho les unes des autres et ont permis une intelligibilité commune des questions ainsi abordées. Chacun avec la méthodologie et la rigueur de sa discipline, Mottez (en sociologie), Cuxac (en linguistique) et Courtin (en psychologie cognitive) ont ouvert des espaces de rencontre entre sourds et entendants (Dalle-Nazebi, 2006).

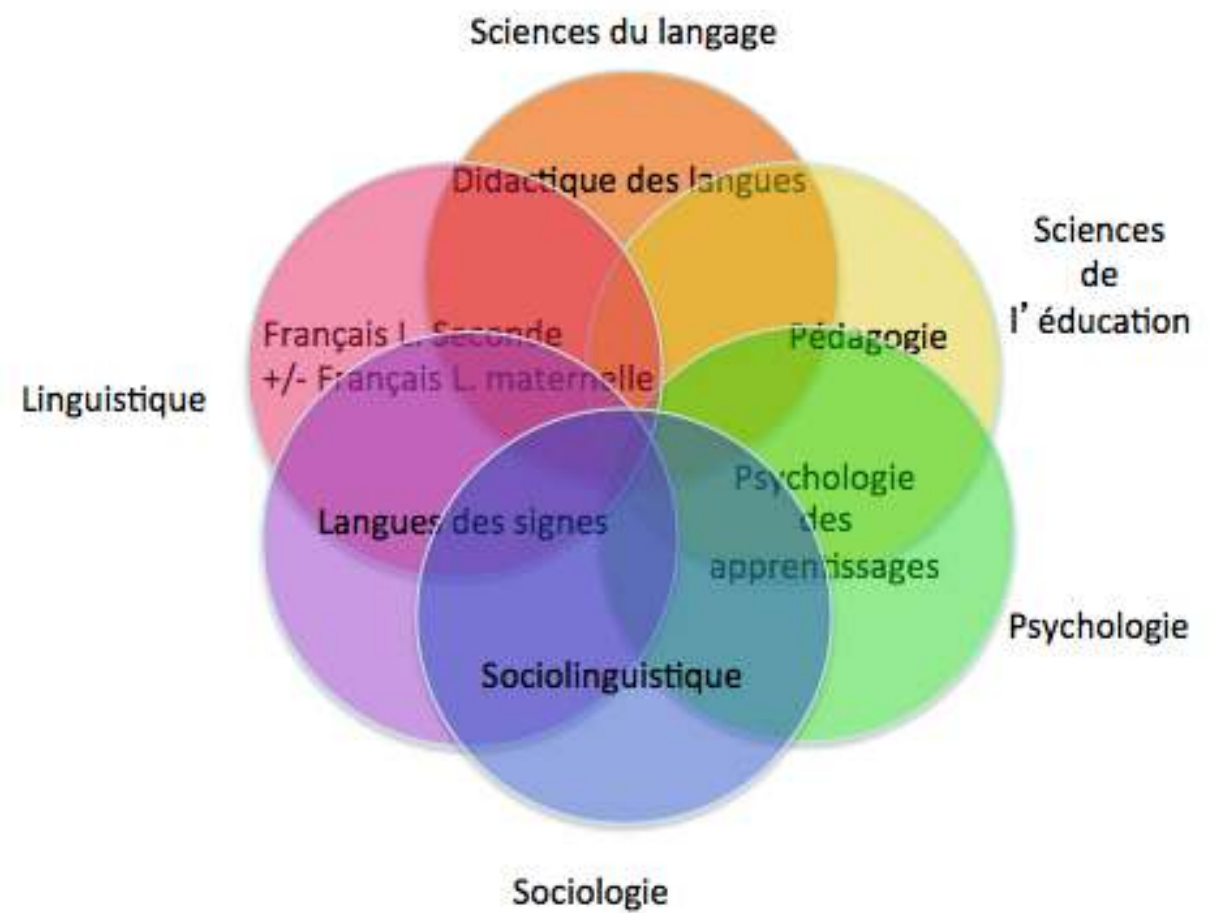

intenant que les recherches sur les sourds sont de plus en plus nombreuses et les champs disciplinaires plus divers, elles cherchent à ne pas s'enfermer dans une seule discipline, évoluent souvent en essayant de croiser plusieurs approches et pourraient donc être interdisciplinaires. Aussi, beaucoup de chercheurs visent une certaine pluridisciplinarité.

Par ailleurs, la question de la représentativité mériterait d'être posée ici, rapidement. Les données chiffrées confondent presque toujours tous les handicaps ce qui peut être un indicateur de l'évolution du regard porté sur eux par la société des « valides » mais ne dit pas grand-chose sur le sort des personnes en situation de handicap, et encore moins sur celui des enfants à besoins éducatifs particuliers.

Quand elles ne présentent pas leurs données tous handicaps confondus, les enquêtes quantitatives superposent le plus souvent aveugles et sourds sous la rubrique " handicaps sensoriels ", afin de répondre à des critères de représentativité et de ne pas raisonner sur des quantités considérées comme négligeables ${ }^{25}$. Quoi qu'il en soit, quand une catégorie « handicap auditif » existe, elle manque de granularité : rien ne distingue qui est utilisateur de la langue des signes ou pas, ni qui suit sa scolarité en inclusion, en établissement médico-social ou dans des classes dédiées en LSF, ce qui constitue un distinguo fondamental et permet d'évaluer tant l'acquisition des compétences de type scolaire, la socialisation que l'estime de soi, pour chacun des dispositifs sans a priori. De surcroît, l'utilisation précoce d'un premier mode de communication chez un jeune

TIPA. Travaux interdisciplinaires sur la parole et le langage, 34 | 2018 
enfant sourd, puis d'un autre à certaines étapes de son parcours, rend aussi très opaque la mesure des atouts de l'oralisme ou de la LSF dans l'éducation ${ }^{26}$.

Les différentes compétences et les besoins des professionnels déjà en place n'ont jamais été vraiment répertoriés, et les formations ont évolué " par amendements successifs " alors qu'une « remise à plat » serait nécessaire. Un vaste champ reste à explorer qui relève à la fois des sciences du langage, des sciences de l'éducation et de l'ingénierie de formation.

\section{Connaître le terrain sans compromettre la neutralité du chercheur}

Les pionniers de la recherche sur le particularisme sourd en France ont proposé un argumentaire de haut niveau tout en ayant l'intelligence et la modestie de laisser les sourds trouver leur place et avancer eux-mêmes. C'est ce que montrent les recherches de Kerbourc'h (2006), de Feldman (2001) et de Mato (2017) en décrivant le rôle crucial de certains universitaires dans l'accompagnement du "réveil sourd». Tous les entendants impliqués dans le milieu sourd n'ont pas eu cette posture, loin s'en faut.

Il pourrait sembler qu'il y ait conflit d'intérêt, en quelque sorte, à parler du rôle des universitaires, étant nous-mêmes auteurs et en position de chercheurs. Toutefois, ce ne sont pas nos propres travaux que nous valorisons ici, mais les travaux antérieurs d'autres chercheurs : nos "devanciers", comme disaient les sourds signeurs à propos des grandes figures de la communauté sourde d'autrefois... S'il fallait renoncer à parler d'eux, au moment où la question d'une éventuelle didactique spécifique nous pousse à observer la place de la LSF et de ses usagers au sein de l'université, leur présence manquerait beaucoup au contraire.

À celui qui se lance dans une recherche sur les sourds, plus encore que dans d'autres champs du travail éducatif ou social, la question du degré d'implication ou de son engagement militant est toujours renvoyée. Faudrait-il absolument être extérieur à la surdité pour ne pas être englué dans l'émotion, au risque d'être un observateur en mirador? Mais que veut alors dire "extérieur»? Les discours des médecins, des psychologues ou des spécialistes de la pathologie du langage qui travaillent sur la surdité sont-ils plus légitimes et donc plus recevables aux yeux des instances décisionnaires? Faut-il au contraire être concerné, soi-même ou de près, par le handicap pour être autorisé à traiter tel ou tel aspect de la question? Mais que veut alors dire "concerné "? Comment prendre en compte les avancées du mouvement Nothing about us without us, né dans les années 1990, concernant leurs choix linguistiques faits pour les sourds en France?

\section{La place des recherches sur les sourds à l'université}

Les langues gestuelles sont des langues naturelles produites par l'activité humaine. On peut leur trouver des similitudes avec d'autres langues, ou faire des liens entre la minorité sourde et d'autres minorités, ou entre les difficultés d'apprentissage de certains enfants sourds et d'autres situations d'apprentissage.

Avec une langue accessible, l'instruction et les capacités de raisonnement, tant sur la langue nationale que dans tous les autres champs disciplinaires abordés dans la scolarité, revêtent des similarités frappantes avec l'enseignement à des élèves tout- 
venants. Ce n'est certes pas une mince affaire, mais cela fait de l'enseignement aux enfants sourds la même affaire que pour tout autre défi éducatif et peu importe alors la capacité de l'enfant à vocaliser distinctement et à lire sur les lèvres de son professeur ou de ses camarades.

Compte tenu du fait qu'utiliser la LSF pour enseigner améliore la compréhension par un enfant sourd de toutes les disciplines scolaires, que l'élève soit en capacité d'oraliser ou pas y compris l'étude du français et l'apprentissage de la lecture ${ }^{27}$, la question de la langue de scolarisation des enfants sourds se situe potentiellement dans les sciences de l'éducation.

\section{Être en conformité avec les conditions d'usage de la langue signée}

En contexte pédagogique, la nature de l'acte éducatif, la relation entre enseigné(s) et enseignant(s) et les pratiques retenues par le professionnel sont à reconsidérer sous un angle nouveau face à un public d'enfants sourds. En effet, quelles langues permettant des activités cognitives et métacognitives, et pouvant être également utilisées de façon intangible dans des entretiens liés au travail social, comme dans le cadre de conférences, de campagnes électorales, d'un journal télévisé ou de débats de l'Assemblée Nationale, devraient-elles se contenter d'une place parmi les langues véhiculaires?

Poser la question de la langue choisie, et faire éventuellement le choix de procéder autrement qu'avec le français oral exclusivement, conduit, du même coup, à envisager la place incontournable des pédagogues sourds dans le paysage éducatif, à s'assurer de la qualité des «nourrices linguistiques » retenues pour enseigner aux enfants sourds et à questionner les modalités d'apprentissage dans des termes distincts des autres langues, minoritaires ou non. Ceci étant posé, les conséquences des choix éducatifs sur la scolarité des sourds, sur la transmission atypique de leur langue première d'une part ou bien sur l'acquisition par des non-sourds d'une LSF "exotique" d'autre part, plaideraient plutôt en faveur d'une didactique qui lui soit dédiée. Cet atypisme des langues signées constitue-t-il une raison suffisante pour créer une nouvelle branche de la didactique des langues et des cultures. Nous reviendrons bientôt sur ce point.

\section{Le tournant du XXle siècle en France}

32 Alors qu'à l'étranger, des postes sont ouverts à des chercheurs sourds depuis les années 1970, le premier poste de «maître de conférence associé à temps partiel » (PAST) réservé à un enseignant sourd signeur n'a pu voir le jour qu'en 2000, à l'Université de Paris 8 en sciences du langage. Avant l'ouverture de tels postes, aucun véritable cursus universitaire en LSF n'avait pu exister, et ce, bien que la langue des signes fût éventuellement un objet d'étude au sein de ces mêmes universités.

Parallèlement, et sans qu'une didactique de la LSF soit encore établie, des formations pionnières se développent en France en s'appuyant sur les réflexions, engagées surtout par les associations du mouvement sourd. C'est au travers de recherches-actions que les chercheurs, les acteurs de l'éducation des sourds et la communauté signante vont pouvoir s'interroger sur la normalisation de cette langue qui n'avait fait l'objet d'aucun enseignement universitaire auparavant et sur son enseignement, en tant que langue 
seconde tout d'abord, puis, un peu paradoxalement, en tant que langue première seulement par la suite.

Alors que depuis une dizaine d'années, la reconnaissance de la LSF semble acquise et que son enseignement a progressé, un certain nombre d'obstacles structurels freinant l'ouverture des classes en LSF subsistent encore. Entre autres, la grande hétérogénéité au sein des classes en LSF, que le projet affiché soit clairement bilingue ou pas, est renforcée par le manque de visibilité de telles structures. Ces classes sont encore plus marginales dans l'enseignement français que les classes en langues régionales. Les familles ne savent pas où porter leur choix ni où il existe des structures correspondantes, si bien que certains élèves sourds arrivent en classe bilingue en cours de cursus, ou après un début de parcours scolaire qui les a placés en situation d'échec, avec un retard langagier (retard en LSF aussi, alors qu'on ne pense souvent qu'au retard langagier en français). Récemment, quelques rares recherches ont émergé également sur le français comme langue seconde ${ }^{28}$ pour les sourds (Hamm, 2010 ; Perini, 2011) ou sur l'acquisition de la lecture dans un enseignement en $\mathrm{LSF}^{29}$.

\section{Un faisceau de réflexions d'ordre épistémologique}

35 L'étude de la LSF et de ses locuteurs, qui plus est quand elle est menée conjointement avec eux, dessine, à la fois, un défi scientifique et un bel enjeu épistémologique, du fait qu'elle exige d'ajuster voire de modifier des concepts déjà établis. Dès le "réveil sourd", les chercheurs intéressés par la langue des signes et les sourds ont cheminé ensemble ${ }^{30}$. La force de cette complicité tient certainement au fait que la surdité se comporte comme un catalyseur efficace de la réflexion : elle questionne autrement des concepts faussement évidents.

\section{Des évidences à reconsidérer}

Celui qui ne connaît pas la surdité est souvent assez perplexe à l'idée que le handicap « tombe » pour tous les protagonistes s'ils utilisent la langue des signes, ce qui n'est pas le cas des autres situations de handicap.

Parallèlement à la question des compétences communicationnelles, analyser les interactions langagières entre sourds oblige à ré-interroger des éléments aussi fondamentaux que ce qu'est une "langue » (qu'elle passe par les sons ou pas), ainsi que ce qui définit la langue dite maternelle et également la langue "première». Que recouvre la «maîtrise » d'une langue, dès lors que les protagonistes sont empêchés dans la conversation?

Le fait de vouloir assurer une scolarité à un enfant sourd exige des réajustements de fond dans l'acte d'enseigner, face au nécessaire choix de la langue pour la modalité orale : jusqu'où un enseignant doit-il accepter d'échanger avec ses élèves dans une langue nécessairement lacunaire, faute de mieux ? Comment peut-il s'appuyer sur une langue confortable, et à quel prix pour l'enfant, mais aussi pour la société ? Que suppose l'utilisation d'une langue gestuelle sans écriture, dans le cadre d'une scolarisation qui doit contribuer néanmoins à construire des compétences de lecteur? Un « enseignant pour sourds » doit-il être sourd lui-même? Que veut dire accompagner les apprentissages d'un élève sourd, si on ne le comprend pas? 

dans le cas des enfants sourds, d'orienter les observations sur le déficit attentionnel vers la flexibilité attentionnelle. La recherche de Leroy a remis en cause l'usage du terme «bilingue » appliqué à certaines pratiques éducatives pour les enfants sourds. Geffroy a mis en évidence une discrimination structurelle dans ses travaux sur l'accès des sourds aux professions de l'enseignement, malgré un mode de recrutement qui se veut non-discriminant, ce qui exige de réfléchir aux conditions de l'inclusion pour ne pas provoquer une exclusion de l'intérieur. Burgat (2009), puis Vanbrugghe (thèse en cours) réexaminent le rôle de la vocalisation et la place de la conscience phonologique dans l'acte de lecture et nous poussent à envisager sous un angle nouveau la compétence du lecteur.

43 Enfin, faire de la langue des signes un objet d'étude, a fortiori du fait que ses enseignants peuvent être sourds signeurs et formés principalement par le milieu associatif, interroge les politiques linguistiques françaises et sert à redéfinir des concepts-phares tels que le plurilinguisme, la diversité culturelle, l'interaction pédagogique, l'apprentissage entre pairs. Rappelons, à ce sujet, qu'un rapprochement est possible entre l'histoire des classes en langues régionales ${ }^{32}$, ce qui inclut les questions de formation des professeurs des écoles en poste dans ces classes et les classes bilingues en $\mathrm{LSF}^{33}$, aujourd'hui toutes reconnues par l'Éducation nationale.

Ces remarques montrent qu'il serait dommage de se priver du regard qu'une didactique spécifique aux LS pourrait apporter.

\section{Prendre en compte un mode de pensée visuel}

45 " Dire en montrant » est inhérent à la surdités ${ }^{44}$, et cette intention sémiotique s'invite spontanément dans une interaction pédagogique conduite par des pédagogues sourds, parce que leur vécu de la surdité leur permet de mobiliser ou de raviver la capacité à 
générer du sens dans une modalité visuo-gestuelle accessible à tous, et à un élève sourd en particulier. Ils adoptent en effet un fonctionnement en analogie avec l'expérience perceptivo-pratique, du fait de repères proprioceptifs spécifiques. C'est pourquoi, les sourds dans les équipes apportent, plus que leur langue, leur connaissance expérientielle de la surdité, ce que ne peuvent pas apporter des enseignants entendants (Courtin, 2002): on constate, entre autres, l'utilisation de stratégies pédagogiques et une posture éducative atypique (Leroy, 2010). Aussi, chaque fois que c'est possible, des éléments d'observation prenant en compte les similarités et les variations entre les dispositifs d'enseignement en LSF avec enseignants sourds ou entendants signeurs, et les autres dispositifs pourraient faire l'objet de recherches approfondies ou avoir une place dans les protocoles d'enquête des chercheurs en sciences de l'éducation.

Au-delà des disparités qui peuvent exister entre différentes langues des signes (microcommunautaires ou individuelles dans le cas des langues émergentes), des chercheurs comme Sallandre, Garcia et Fusellier ont mis en évidence l'originalité de la conception (imagée) et l'appréhension (visuelle) du monde qu'ont les sourds, capables de coconstruire spontanément une langue visuo-gestuelle riche avec leurs proches pourvu que ceux-ci se montrent réceptifs. Rien qu'en observant l'émergence d'une langue naturelle chez un locuteur sourd n'ayant fréquenté aucun signeur, ni à l'école ni dans son entourage $e^{35}$, on entrevoit combien nous aurions à apprendre de la pédagogie propre aux sourds.

Grâce à la prise en compte du mode de pensée des sourds, une authentique didactique de la LSF nourrirait donc aussi la recherche en sciences de l'éducation, puisqu'avec la présence de la LSF dans l'enseignement (bien plus qu'au travers des seuls cours de LSF) se développe une véritable centration de la pédagogie sur le sens. Ce serait aussi aller dans le sens d'un regard sur les personnes dites handicapées comme étant « autrement capables ", nous apprenant à co-construire du sens au-delà de la seule activité verbale (vocale ou signée). Des recherches approfondies pourraient ainsi avoir un certain retentissement sur la scolarisation de tous les autres publics et sur la formation des professionnels.

\section{De l'intérêt didactique de l'étude des langues signées}

Nous adoptons pour principe que le rôle de toute didactique est d'observer des faits d'enseignement et éventuellement de construire des concepts capables de les décrire.

Dans le cas de l'acquisition d'une langue étrangère, on observe la construction d'un lecte d'apprenant, surtout dans les premiers temps. Même si l'acquisition se fait dans ce qui s'apparenterait à l'immersion et au plus jeune âge, une interlangue transitoire assez similaire pourra perdurer d'autant plus longtemps pour quelqu'un dont l'audition est défaillante. Malgré la rééducation orthophonique, la construction d'un lecte de la langue de l'école sera perpétuelle pour un enfant sourd, quand bien même l'utilisation de cette même langue est intensément stimulée en famille.

Dans d'autres pays, ces questions ont débouché sur la création de deaf studies. L'initiative est venue, au milieu des années 1980, de Padden et Hemphris, tous deux sourds et chercheurs en anthropologie à Gallaudet, ce grand campus de Washington offrant depuis longtemps la possibilité aux sourds, signeurs ou non, de suivre une scolarité en langue des signes de la maternelle à l'université (Dalle-Nazebi, 2006). L'idée 
d'un Centre de deaf studies a essaimé au fil des rencontres et des colloques. Elle s'est structurée assez vite en Allemagne (à Hambourg) et au Royaume-Uni (à Bristol). En France, elle n'a pas pris, mais a influencé les représentations des personnes sourdes sur elles-mêmes ainsi que ceux qui accompagnaient le « réveil sourd » parti, rappelons-le, d'un voyage à Gallaudet organisé par Mottez à la fin des années $1970^{36}$. Autour des deaf studies se retrouve un microcosme de chercheurs et d'acteurs du milieu sourd qui se situent dans une approche socio-anthropologique, tout en étant liés de plus loin aux autres spécialistes de chaque discipline représentée.

51 Il faut noter que dans les pays scandinaves, la surdité relève principalement du champ des disability studies, parce que située dans le champ du handicap. Or, la LS étant reconnue et l'accessibilité dans tous les moments de la vie étant assurée, la présence des sourds dans la recherche s'en trouve banalisée, si bien que gens de terrain et chercheurs trouvent à se nourrir réciproquement. Cette perspective, plus socioanthropologique, constitue le versant non pathologique de la surdité et positionne d'emblée les sourds comme des personnes "autrement capables", en pleine adéquation avec les recommandations internationales actuelles.

Nous n'en sommes pas à en France ${ }^{37}$, où une LS ne peut pas être vue comme l'un des éléments constitutifs d'une culture propre aux sourds, même depuis que la LSF a droit de cité. Les représentations sociales à l'égard des locuteurs sourds, comme personnes handicapées avant tout, établissent une différence fondamentale parce qu'elles colorent complètement le regard porté sur un élève sourd et, de fait, le traitement de la question des sourds, tant sur le terrain des politiques éducatives et linguistiques que sur celui de la recherche.

\section{Où situer la LS dans la didactique?}

53 Alors qu'elle existe depuis des siècles, la langue des sourds est avant tout une langue « de tradition orale ${ }^{38}$ » qui, avec des périodes moins fastes que d'autres, s'est transmise au sein des écoles spécialisées, et presqu'exclusivement hors des temps de classe. Enfants et adultes sourds ne s'étant vu accorder au mieux qu'un simple droit d'usage, cette langue évoluera sans grille d'évaluation jusqu'au début du XXI ${ }^{\mathrm{e}}$ siècle, avec la création en 2002 d'un référentiel sur le principe du Cadre européen Commun de Référence pour les langues.

Comme d'autres langues de tradition orale, l'étude de la LSF aurait pu se contenter d'une place au sein des sciences du Langage, si ce n'est que, dans ce champ, dès lors qu'une langue est enseignée et qu'elle devient même langue d'enseignement, ses locuteurs mais aussi ceux qui l'apprennent ont envie et besoin de penser sa propre didactique. Pour ce faire, il avait fallu dans un premier temps décrire la LSF, quitte à rendre compte de la "phonologie", du lexique et de la syntaxe d'une langue gestuelle avec des outils comparables à ceux utilisés pour les langues vocales. Mais la réflexion des principales associations du "réveil sourd " s'était engagée non seulement vers la diffusion de la LSF, mais aussi immédiatement vers la construction de savoirs de type scolaire par le biais de cette langue ${ }^{39}$. En discutant des apprentissages via une langue gestuelle, le débat s'est très vite orienté vers la lutte contre la sous-instruction dans laquelle sont confinés tous ceux qui ne peuvent pas interagir avec une langue audiovocale, nous l'avons rappelé plus haut. Depuis, les pédagogues sourds appellent avec insistance les chercheurs à créer un champ didactique consacré à la LSF, afin d'entrer 
ensuite avec eux dans ce qui pourrait bien être un cercle vertueux, sans oublier que tout en ayant des degrés différents d'implication, chacun apporte une vision complémentaire des mêmes phénomènes. Mais la didactique des langues et des cultures tarde à prendre la LSF en considération, autrement que de façon marginale. La thèse de Morillon en 2001 n'a pas eu beaucoup d'écho. Il a fallu attendre les travaux de Mugnier en 2006 et de Leroy en 2010 pour que la relation enseignant/apprenant soit analysée sous ses aspects didactiques en tenant compte de l'usage de la LSF. Alors, ne serait-il pas dommage de ne pas fédérer toutes les recherches, portées par des chercheurs avérés ou potentiels venus d'horizons différents? Rien que pour cette raison, il serait temps d'engendrer une didactique dédiée aux LS.

La question de l'éducation des sourds peut ne pas s'enfermer dans la seule question du fonctionnement ou de la maîtrise de la langue, du seul fait que la linguistique convoque souvent d'autres disciplines. Ainsi, certaines études se sont intéressées, petit à petit, au versant praxéologique: observation des pratiques, modalités de transmission d'une langue a priori originale par des sourds qui en faisait finalement leur métier, identification des contextes d'utilisation de la LSF, analyse de l'action et formations pour l'action. Autrement dit, bien que les pionniers du bilinguisme dans l'éducation des sourds aient souhaité à la fois des formations pour les nouveaux enseignants depuis peu sur le terrain et pour les futurs pédagogues sourds, l'ancrage universitaire n'a pas été initié dans le champ des Sciences de l'éducation.

Entre la toute première proposition de formation professionnelle d'enseignants accessible aux sourds, conçue par l'association 2LPE et présentée au ministère de l'Éducation nationale en 1982 (puis transformée par le CNEFEI de Suresnes en 1986 pour former des " experts en LSF » sourds), et la première Licence professionnelle ouverte en 2004, d'autres formations associatives avaient vu le jour. Citons entre autres le DPCU «spécialisation enseignement de la LSF » créé par l'association Visuel-LSF en 2000 en partenariat avec l'université Paris 8. On notera que la licence professionnelle intitulée "Enseignement de la LSF en milieu scolaire", ouverte en 2004 et portée par la Formation permanente de l'université de Paris 8, est le premier cursus universitaire dédié au public signant.

Cette formation avec l'intégralité des enseignements dispensés en LSF - ou interprétés - et la possibilité de rendre des travaux en LS-vidéo, a ainsi offert une formation professionnelle et diplômante aux enseignants sourds signeurs et, par la même occasion, une reconnaissance pour pouvoir exercer en milieu scolaire. Toutefois, sur le terrain de l'éducation, les acteurs sourds, bien qu'indispensables, peinent toujours à occuper la place qu'ils méritent et s'épuisent à la tâche, sans pouvoir mutualiser leurs travaux ni se coordonner. C'est ce qui ressort des différents rassemblements associatifs ou les différentes journées d'études du milieu sourd: universités d'été de 2LPE, colloque «LSF : enseignement, identité professionnelle et programme » en octobre 2006, journées de l'AFFELS, rassemblement sur l'éducation bilingue (INS-HEA, automne 2016), etc.

En juin 2006, la LSF a été intronisée comme discipline optionnelle possible au baccalauréat. Un programme officiel, une dotation horaire et un recrutement d'enseignants certifiés ont été mis en place entre 2008 et $2010^{40}$. Voilà qui pousserait à établir un constat encourageant. Mais ces mesures appliquées d'abord dans l'enseignement secondaire ordinaire concernent donc majoritairement des élèves entendants, tandis que très peu de classes en LSF existent et que les élèves sourds, eux, 
en ont tiré peu de bénéfice en n'ayant quasiment pas de cours portant sur cette langue. Un nouveau paradoxe est en train de voir le jour: les parents tout-venants sont très enthousiastes à l'idée que leurs enfants apprennent la LSF et qu'un cadre leur permette de la choisir comme langue optionnelle, convaincus que cela leur apportera et pourra toujours être utile, alors que dans le même temps, les parents d'enfants sourds assurent une lutte permanente pour que leurs enfants et eux-mêmes aient un accès facilité et moins parcimonieux à cette langue si cruciale pour eux ${ }^{41}$. C'est pourquoi Meynard (2013 : 34), entre autres, propose de rester en alerte car il ne faudrait pas « hélas penser que la sombre destinée réservée à la LSF appartient désormais à un passé révolu dont nous serions définitivement sortis ".

Certes, les formations et la recherche universitaires avancent. De plus en plus d'étudiants sourds ont accès aux études supérieures et réfléchissent à la manière d'avoir accès au contenu des enseignements en $\operatorname{LSF}^{42}$ et ce, malgré le manque de moyens pour bénéficier totalement d'une interprétation en LSF. Paradoxalement, peu d'enfants sourds ont accès aux rares classes bilingues LSF/français écrit permettant un enseignement de "qualité » en LSF (seulement 1 à $2 \%$ des élèves sourds, selon l'Association Nationale des Parents d'Enfants Sourds ou ANPES ${ }^{43}$ ).

Le rattachement indécis des travaux portant sur la langue des signes est aussi le reflet du statut de la langue et de ses locuteurs. La question du recrutement des enseignants de LSF n'a été résolue que très partiellement par la création d'un concours de l'Éducation nationale, le CAPES de LSF depuis 2010, fait marquant symboliquement.

61 Pourtant, les besoins du terrain sont considérables mais mal chiffrées, si bien que les professionnels en poste ou en formation cherchent des repères auprès des universités et ce dans un réseau européen, voire international, puisque les LS ont cette ouverture et cette facilité d'interaction.

\section{Complicité entre le terrain et la recherche}

En général, toute didactique part du terrain occupé par les praticiens, puis ces derniers font une place aux didacticiens dans leur pratique, et les sollicitent dans leurs formations. L'émergence récente de la didactique du Français Langue Étrangère (FLE) est un exemple assez caractéristique de ce processus : l'entrée du FLE à l'université et la professionnalisation du métier de professeur de FLE se sont produites dans les années 1980 , en s'appuyant sur deux organismes, essentiellement issus du terrain, le Bureau d'enseignement de la langue et de la civilisation françaises à l'étranger (BELC) ${ }^{44}$ et le Centre de recherche et d'étude pour la diffusion du français (CREDIF) ${ }^{45}$.

Avec la LSF, une rhétorique inverse s'est installée qui ne partirait pas du terrain pour contribuer à la naissance de la didactique. Dans un premier temps, des études et des recherches se sont ouvertes aux sourds décidés à reconquérir le terrain. Des conférences ou des formations dédiées ont été enfin accessibles, encouragées par les travaux de chercheurs encore marginaux. Ces derniers se sont beaucoup déplacés pour soutenir les initiatives isolées du terrain associatif.

Reconnaître la compétence des praticiens a permis de justifier dans un second temps la mise en place de qualifications pensées pour eux (Kerbourc'h, 2006; Leroy, 2010; Geffroy, 2015). Les premières formations associatives dans le domaine de l'enseignement de la langue des signes (pour des professionnels sourds ou pour les familles), ou dans le domaine de l'interprétation pour les professionnels entendants et 
non signants, ne s'appuyaient sur aucune antériorité : les gens de terrain tâtonnaient et les spécialistes pouvant assurer un appui théorique étaient rares. Puis, un va-et-vient s'est enclenché petit à petit entre praticiens et didacticiens.

Dans les débats quant aux choix de société transversaux autour de l'école inclusive, chercheurs en LSF et professionnels de la surdité se montrent très circonspects s'agissant de l'inclusion des élèves sourds. En effet, si repenser l'équilibre entre classes d'inclusion et classes dédiées doit contribuer à une différentiation raisonnée, l'impact crucial du choix de la langue d'enseignement peut compromettre la mission principale de l'école : construire des apprentissages à hauteur de leurs capacités pour les enfants sourds à l'instar de tous les autres.

Ceci étant posé, loin d'être un repli sur la communauté sourde et sur sa langue de prédilection, la didactique de la LSF ferait place à la diversité des points de vue, y compris des recherches portant sur les représentations du handicap en général et d'autres qui, elles, se penchent sur les politiques éducatives ou linguistiques. Sourds signeurs et entendants y travailleraient ensemble, l'accessibilité serait bien mieux assurée (et systématiquement prévue) que partout ailleurs.

\section{Didactique et bilinguisme sourd}

67 Contrairement à la didactique des LV, une didactique de la LS exige de penser non seulement l'enseignement de cette langue, mais aussi l'approche particulière nécessaire aux autres disciplines parce qu'enseignées en LS, l'articulation idéale étant le bilinguisme dans la pédagogie.

68 Certes, la construction d'une didactique de la LSF renvoie à la question de la standardisation de la langue, et les récents programmes du Ministère de l'Éducation nationale nous font prendre conscience que la LSF n'échappe pas à la contrainte de la norme. Toutefois, ne pourrait-on pas aujourd'hui envisager de faire à cette langue une place basée sur l'usage de ses locuteurs et de la communauté signante? Ainsi, elle serait non plus un bel objet d'étude atypique mais bien une langue à part entière, contribuant à faire reconnaitre en même temps les droits de toute personne sourde communiquant en langue des signes, quels que soient son âge, son parcours de vie et sa place dans la vie sociale.

69 Même si créer un champ propre à cette langue et à la communauté qui la parle aurait des incidences profondes sur la formation des professionnels de l'éducation, cela n'impose pas une vision focalisée sur la surdité. Les avancées issues de la réflexion sur l'éducation des sourds peuvent irradier jusque dans les adaptations pédagogiques et contribuer à mettre en œuvre une école plus inclusive qui n'ait plus le cas des sourds comme préoccupation, ni même un traitement des élèves par catégorie. Cela suppose aussi que les différents travaux circulent jusque dans les formations de professeurs.

70 Il faudrait un article entier pour aborder le problème des plans de formations et des contenus à destination des professionnels de l'enseignement bilingue pour les sourds. Nous n'en ferons ici qu'une présentation rapide :

71 - La préparation au CAPEJS (certificat pour enseigner dans les établissements du Ministère de la Santé) à Chambéry a été ouverte aux sourds depuis longtemps. Mais les enseignants bilingues (et tout particulièrement les enseignants sourds) n'y trouvent 
pas leur compte du fait que la formation proposée n'envisage pas spécifiquement l'enseignement en LSF des différentes disciplines aux élèves sourds.

- L'INS-HEA de Suresnes (le centre de formation pour les enseignants spécialisés de l'Éducation nationale), propose un cursus conduisant à la "spécialisation ", alors que les enseignants des classes bilingues se voudraient plus proches des enseignants "ordinaires", si ce n'est qu'ils utilisent une autre langue d'enseignement. La préparation au Certificat de spécialisation et les stages de formation continue sont réservés aux seuls enseignants déjà titulaires, ce qui laisse peu d'espoir aux sourds, souvent contractuels ou dans des situations encore plus précaires ${ }^{46}$.

De plus, il faut tenir compte des fluctuations de l'Éducation nationale dans ses prises de position au sujet de l'enseignement bilingue auprès des élèves sourds. À titre d'exemple, comparons deux BO, celui du 4 septembre $2008\left(n^{\circ} 33\right)$ et celui du 24 juin $2010\left(n^{\circ} 25\right)$, sur trois points :

$\left.741^{\circ}\right)$ Le statut pédagogique de la LSF : le document de 2008 posait clairement la maitrise de la LSF comme préalable aux autres apprentissages, tandis que celui de 2010 revenait à une approche défective en mettant les difficultés de lecture des sourds sur le compte de «la quasi-impossibilité d'avoir recours au code de correspondance graphophonétique $\aleph^{47}$. Aucune de ces deux approches n'est vraiment basée sur des résultats de recherche, ni sur l'étude des politiques sociales et linguistiques adoptées par d'autres pays qui nous donnent à voir d'autres postures éducatives quant au handicap et quant à leurs langues minoritaires - dont la langue signée locale ${ }^{48}$.

$2^{\circ}$ ) Les pôles LSF : le document de 2008 les conçoit comme des «parcours bilingues en $L S F$ ». Ils n'existent plus en tant que tels, puisque le second document, en les renommant Pôles d'accompagnement à la scolarisation des élèves sourds (PASS), élargit considérablement leur mission à l'ensemble des "dispositifs pédagogiques et technologiques permettant à tous les jeunes sourds, quel que soit le mode de communication choisi par leurs familles, de suivre un enseignement au plus près possible d'une scolarisation ordinaire sans se focaliser sur la seule LSF ». Avec la création du poste plutôt ambivalent de médiateur pédagogique $e^{49}$, (à la fois enseignant formé, interprète confirmé, et ayant une formation approfondie sur la prise en charge des élèves en difficulté), quelle place les enseignants sourds peuvent-ils avoir pour apporter des réponses aux acteurs du terrain? Qu'en est-il aujourd'hui depuis la création des PEJS ${ }^{50}$ (Pôle d'enseignement pour jeunes sourds)?

$3^{\circ}$ ) La notion de bilinguisme enfin : parler à nouveau dans le BO n 25 de 2010 de "mode de communication" ou de "communication bilingue" au lieu d'éducation bilingue, terminologie employée dans le BO de 2008, entretient la confusion. On constate sur le terrain, dans le cas d'élèves rejoignant un dispositif bilingue où leurs camarades ont suivi le cursus depuis les classes primaires, les difficultés rencontrées par eux-mêmes comme par les enseignants.

77 La réalité du terrain montre souvent un enseignement aux sourds assuré en langue française, avec le recours d'une LS véhiculaire et, quand c'est possible localement, une ou deux heures hebdomadaires de cours de LSF. La logique de l'inclusion généralisée vers laquelle s'oriente la politique de scolarisation des enfants sourds, au même titre que pour tout enfant dit à Besoins éducatifs particuliers, conduit à une individualisation des réponses. Le principe de différentiation n'est pas condamnable en soi, mais appliqué ainsi, il néglige une adaptation pédagogique majeure qu'envisage la 
pédagogie bilingue: construire les apprentissages avec une langue d'enseignement accessible et utilisée dans un groupe de pairs.

Dans le cadre d'une didactique de la LSF constituée, les choix éducatifs se feraient sur des critères plus objectifs que passionnels, en connaissance de cause. L'observation des spécificités de l'enseignement aux enfants sourds nous offre une belle occasion de mettre en œuvre le principe qui veut s'attacher aux besoins de l'apprenant ${ }^{51}$ pour le faire avancer dans ses acquisitions, plutôt que de maintenir un enseignement " descendant ». C'est pour mieux répondre à ces besoins que l'enseignant doit chercher à comprendre et à mieux respecter le mode d'apprentissage des apprenants sourds - et des enfants sourds en particulier - tout en intégrant leur langue et leur culture. En général, une telle approche vise l'émancipation cognitive de l'enfant: "Or la pédagogie, c'est précisément le fait de récuser, tout à la fois, l'enseignement programmé, qui réduit l'être à une machine apprenante, et la mise en place d'enclaves libertaires, où l'on attend passivement la merveilleuse éclosion d' "aptitudes innées". Le pédagogique, c'est l'effort pour échapper à ces deux dérives-là et construire des situations qui sont, à la fois, vraiment structurées, avec des médiations culturelles fortes, et qui accompagnent l'émergence d'un sujet. "Faire pour que l'autre fasse", voilà, depuis Rousseau, la maxime de la pédagogie » (Meirieu, 2009).

\section{Et si nous tentions de définir la didactique de la LSF ?}

Une didactique de la LSF en est peut-être à ses balbutiements. Son histoire s'est écrite, à l'instar de celle de la reconnaissance de cette langue, à partir du travail des associations nées avec le «réveil sourd" et des premiers pédagogues bilingues ${ }^{52}$. Le versant recherche s'est constitué autour des premiers travaux universitaires ayant accompagné ce mouvement depuis les années 1980, puis à travers les mémoires universitaires situés dans la didactique des langues et des cultures, mais axés spécifiquement sur la LSF : Munoz (DEA en 1997 à Paris 5), Morillon (thèse en 2001), Geffroy (DEA en 1999), Hugounenc (DEA en 2003 qui se situait en anthropologie sociale mais partait de l'observation participante d'un cours de LSF), Mugnier (thèse en 2006), Leroy (thèse en 2010). Et on compte un certain nombre d'autres thèses soutenues depuis, ou en cours.

Un travail didactique structuré semble maintenant tout aussi incontournable que l'étude linguistique de la langue elle-même et il peut s'articuler entre ceux qui cherchent à décrire cette langue, ceux qui en observent les usages entre utilisateurs, ceux qui la transmettent, pensent des programmes, conçoivent des progressions pédagogiques ou créent des supports, et ceux qui l'apprennent soit en immersion, soit avec un professeur. Or, dans le paysage scolaire actuel, les classes bilingues touchent moins de $2 \%$ des enfants sourds et subissent de fréquentes remises en cause, ou menacées de fermeture.

81 Néanmoins, que l'enseignant partage la langue des signes avec les élèves sourds ne règle pas la question des enseignements disciplinaires. Une didactique de la LSF devra donc se consacrer aussi aux spécificités des enseignements disciplinaires pour les élèves sourds, en particulier dans le cadre d'une pédagogie bilingue. Par ailleurs, en dehors des classes bilingues, l'enseignant face à eux est le partenaire principal dans l'acquisition de compétences communicationnelles, pragmatiques et socioculturelles que les enfants entendants, eux, ont mobilisées très jeunes en acquérant leur langue maternelle, ailleurs qu'à l'école. 
82 Par exemple, comme l'enfant sourd n'a l'expérience d'une communication naturelle efficace que dans une modalité visuo-gestuelle, collaborer entre apprenants et savoir travailler à plusieurs sont des compétences sociales que les enfants sourds peuvent construire, sans entrave, bien mieux en présence d'une LS partagée par tous qu'en milieu ordinaire en raison de la perturbation des interactions, effet collatéral de la surdité.

De tels aspects pédagogiques ne seront pas négligés s'il existe une didactique spécifique de la LSF et si des échanges entre praticiens sourds ayant une connaissance expérientielle de la surdité et praticiens entendants permettent de penser la complémentarité entre la dimension interactionnelle propre aux sourds et les apprentissages (Geffroy, 2015). C'est peut-être sur ce point qu'une société véritablement inclusive va devoir faire ses preuves.

Les premiers projets de classes bilingues s'étaient construits sur le principe des classes en langues régionales. Les actuelles classes bilingues ne renient pas cette filiation. Que ce soit dans l'enseignement en classes bilingues pour sourds ou dans l'enseignement en classes en langues régionales, on note qu'il y a un effet de ricochet entre une transmission atypique de la langue (régionale donc, ou signée) et l'utilisation de cette même langue pour enseigner, car elle devient, dans les deux cas, langue d'enseignement en ne l'ayant pas été auparavant.

Un enseignement bilingue tenant compte des spécificités de la langue gestuelle aide à penser la construction de concepts disciplinaires et de compétences sans se focaliser sur les aspects lexicaux. Si l'enrichissement langagier entraîne le plus souvent un saut qualitatif dans le raisonnement, l'amélioration des acquisitions doit aussi être recherchée sans qu'elles soient encapsulées par les mots. En effet, peu importe que les « signes-mots » de la LSF (Cuxac \& Antionoro Pizzuto, 2010) n'étiquettent pas le monde à la façon de certaines nomenclatures établies pour rendre compte de la terminologie de la discipline (Garcia \& Derycke, 2010), puisque l'empan langagier est plus large en LSF que dans les langues vocales (Cuxac, 2007). Une des adaptations majeures que les enseignants doivent mettre en œuvre consiste à étayer les acquisitions par des stratégies pédagogiques autres que verbales uniquement pour ces élèves sourds.

C'est pourquoi les formations dont bénéficient tous les enseignants, spécialisés ou non, tant dans leurs contenus que dans les modalités de travail, doivent absolument être accessibles à tous, même sourds. On constate que dans toutes les formations auxquelles les pédagogues sourds prennent part, ils étudient et mutualisent leurs pratiques professionnelles $^{53}$. Cela se déroule donc dans une sorte d'entre-soi qui leur est, paradoxalement, souvent reproché. Ouvrir toutes les formations à visée didactique rendrait ainsi moins marginal cette pédagogie de et en LSF. De même, les sourds qui confirment leur vocation de pédagogues au fil de leur parcours de formation peuvent assurer le rôle de nourrice et référent linguistique en proposant une forme d'immersion en LSF tout en enseignant d'autres contenus disciplinaires et ce, sans en faire un perfectionnement linguistique. En effet il s'agit là d'une sorte d'approfondissement cognitif, où les concepts mobilisés sont explicités dans une modalité visuo-gestuelle. Les pionniers de l'enseignement bilingue l'ont presque tous fait, dès le début, sans y avoir été formés, et ont d'ailleurs créé leurs propres supports pédagogiques. La réflexion s'est poursuivie depuis, et enrichie.

87 Enfin, la langue est indissociable de la communauté qui l'utilise. Pourtant, l'idée d'une culture sourde et d'une pensée visuelle est encore souvent contestée, si bien que le lien 
entre langue et culture prend une coloration particulière dans le cadre de la surdité, même maintenant que la LSF a droit de cité. Entre autres, alors que la didactique d'une langue vocale encourage la participation des locuteurs natifs, ce n'est pas sans réticences que le système français s'assure de la participation de personnels sourds. Il y a, bien sûr, des raisons structurelles - qui devraient pouvoir s'atténuer au fil du temps - du fait que suivre une scolarité jusqu'au baccalauréat est un véritable parcours $\mathrm{du}$ combattant pour les élèves sourds. Les bacheliers sourds sont donc rares et seulement 3 à $5 \%$ d'entre eux poursuivent des études supérieures ${ }^{54}$. Ils sont, de ce fait, peu nombreux à pouvoir se présenter aux recrutements d'enseignants des dispositifs communs ou à s'engager dans des parcours universitaires poussés. (Garcia et Burgat, 2016)

Finalement, même si la taille de la population concernée paraît dérisoire puisque les enfants sourds représentent environ $2 \%$ des élèves en situation de handicap ${ }^{55}$, les recherches sur l'enseignement de la LSF et/ou avec la LSF se trouvent confortées par rapport à l'enjeu de taille que représente l'égalité des chances à l'école, en la déclinant pour les sourds, sans oublier que cela peut ouvrir à une meilleure prise en compte des besoins de tout autre enfant dans sa scolarisation.

\section{Conclusion}

Après une quinzaine d'années (soit entre 1975 et 1990) de dévoilement, d'espoir, de découragement, et quelques coups de force même, la langue visuo-gestuelle, cette langue dont la nature-même reflète le mode de pensée des sourds, aura droit de cité en France. Grâce à Markowicz et à Mottez, un premier recueil de textes permettra de faire le point sur l'état des recherches à propos des langues des signes et de les faire connaître $^{56}$, conduisant à considérer, dès lors, les sourds autrement que par le filtre du handicap et de l'anormalité. Les militants les plus engagés tenteront de défendre l'idée encore inconcevable que la surdité ne relève plus uniquement du domaine du soin ni de la rééducation, et que leur langue peut être d'une part objet d'études et, d'autre part, langue de scolarisation.

À défaut d'avoir trouvé une place en didactique ou en sciences de l'éducation, l'étude de la LSF s'est enracinée en linguistique, même pour des travaux plus périphériques portant sur les formations d'interprètes et la traductologie ou sur la pédagogie bilingue. Dans le même temps, les recherches tournées vers la description et l'usage de cette langue visuo-gestuelle ont remis en cause certains postulats en linguistique, notamment.

De nos jours, le statut des enfants sourds à l'école, le statut des enseignants sourds et le statut de la langue des signes portent encore les traces de représentations éducatives happées, implicitement mais en permanence, par une référence à l'image de l'enfant entendant vis-à-vis de la langue française, ainsi qu'à l'archétype de l'adulte chargé d'enseigner d'abord dans une modalité audio-phonatoire. La présence, dans l'éducation des sourds, d'intervenants très majoritairement entendants, se trouvant eux-mêmes handicapés dans leur mission ou envisageant encore la LSF comme une «langue prothèse dans une prescription de dernier recours dans le traitement du handicap " (Millet, 1999 : 111), contribue à l'inertie face à un changement possible de paradigme.

En 1993 déjà, Mottez résumait ce que nous avons voulu aborder ici : 
Si cette pédagogie muette peut être qualifiée de typiquement sourde, qu'est-ce à dire? Qualifier de typiquement sourdes certaines aptitudes, ne signifie pas nécessairement que celles-ci fassent radicalement défaut aux entendants. Il se trouve seulement que, fonctionnant sous d'autres modalités et d'autres contextes, les entendants ne les ont pas assez développées. Qualifier de typiquement sourdes certaines façons de faire, ne signifie pas que les entendants ne puissent à l'occasion procéder spontanément de la même manière ou a fortiori qu'ils ne puissent s'en inspirer ou se les approprier. Ils peuvent les apprendre. Lorsqu'on parle aujourd'hui de pédagogie à propos des intervenants sourds, on ne pense il est vrai qu'à celle que devraient leur enseigner des spécialistes - entendants - pour qu'ils deviennent de bons enseignants. C'est pourquoi j'insiste sur celle qu'ils pourraient peut-être, eux aussi, apprendre aux entendants. (Mottez, in Benvenuto 2006, p. 175) 


\section{BIBLIOGRAPHIE}

\section{Ouvrages et articles}

BIAP (Bureau International d'Audiophonologie) (2003) Le bilinguisme dans l'éducation et l'enseignement de l'enfant sourd. Recommandation 17/3 [en ligne], Corfou. Disponible sur : http:// www.biap.org/fr/archives/72-ct-17-communication-/47-recommandation-biap-173-lebilinguisme-dans-leducation-et-lenseignement-de-lenfant-sourd (consulté le 30 juillet 2018).

Burgat, S. (2009) Un exemple atypique d'acquisition du langage écrit en contexte bilingue : l'enfant sourd locuteur de la langue des signes qui apprend à lire et à écrire en français, in Colloque Jeunes Chercheurs en Acquisition du Langage, Université de Lyon, les 3 et 4 décembre 2009.

Buton, F. (2009) L'administration des faveurs. L'État, les sourds et les aveugles (1789-1885), Presses universitaires de Rennes, p. 311 (Histoire).

Coste, D. (1992) Linguistique de l'acquisition et didactique des langues : repères pour des trajectoires, in Bouchard, R., Billiez, J., Colletta, J.-M., De Nuchèze, V. \& A. Millet (dir.) Acquisition et enseignement apprentissage des langues, Grenoble: Université Stendhal, p. 319-328

Courtin, C. (2002) Actes du colloque « Conceptualisation et surdité » de mai 2001, La nouvelle revue de l'AIS, 17, 1er trim, Suresnes, p. 181-195.

Courtin, C., Limousin, F. \& A. Morgenstern (2010) Évaluer les compétences linguistiques des enfants en langue des signes, une expérience pionnière [en ligne], Language, Interaction and Acquisition, John Benjamin Publishing Company, p. 129-158. Disponible sur : https:// halshs.archives-ouvertes.fr/halshs-00531659 (consulté le 30 juillet 2018).

Cuxac, C. \& E. Antinoro Pizzuto (2010) Émergence, norme et variation dans les langues des signes : vers une redéfinition notionnelle [en ligne], Langage et société, 131, 1, 37-53. Disponible sur : doi:10.3917/ls.131.0037 (consulté le 30 juillet 2018).

Cuxac, C. (2007) Une manière de reformuler en langue des signes française [en ligne], La linguistique, 43, p. 117-128. Disponible sur : doi:10.3917/ling.431.0117 (consulté le 30 juillet 2018).

Cuxac, C. (2000) La langue des signes française : les voies de l'Iconicité, Faits de Langues, 15-16, Paris: Ophrys.

Dalle, P. (2005) Histoire et philosophie du projet bilingue. L'ANPES et le rôle des parents [en ligne], Enseigner en LSF, La nouvelle revue de l'AIS, Hors série 2005. Disponible sur : http:// anpes.free.fr/Doc/NRAIS_HS05-Dalle.pdf (consulté le 30 juillet 2018).

Dalle-Nazebi, S. (2006) Chercheurs, Sourds et Langue des Signes. Le travail d'un objet et de repères linguistiques, Thèse de doctorat en sociologie, Université Toulouse 2 - Le Mirail.

Ebersold, S. (2013) INS-HEA, séminaire du 27 mars 2013, sur « L'orchestration de l'accessibilité à l'enseignement supérieur ».

Feldman, N. (2001) La position des professionnels entendants dans l'association 2LPE, Mémoire de Maîtrise de Sociologie mention Anthropologie, Université Paris 8 Vincennes-Saint-Denis, Paris, $143 \mathrm{p}$.

Fusellier-Souza, I. (2001) La création gestuelle des individus sourds isolés. De l'édification conceptuelle et linguistique à la sémiogénèse des langues des signes, AILE (Acquisition et Interaction en Langue Étrangère), 15, Paris, p. 61-96. 
Garcia, B. \& M. Derycke (2010) Introduction [en ligne], Langage et société, 131, 1, p. 5-17. Disponible sur : doi:10.3917/ls.131.0005 (consulté le 30 juillet 2018).

Garcia, B. \& S. Burgat (2016) Évolution institutionnelle et sociale de la Langue des Signes Française (LSF) et de ses locuteurs sourds : place et rôle de l'Université, in Hélot, C. \& J. Erfurt (eds) L'éducation bilingue en France : politiques linguistiques, modèles et pratiques, Lambert-Lucas, p. 332-346.

Geffroy, V. (2015) La formation des pédagogues sourds. Étude exploratoire. Thèse de doctorat en didactique des langues et des cultures, Université de Paris 8/Vincennes-Saint-Denis.

Grosjean, F. \& H. Lane (1979) Psycholinguistique et langue des signes, in Langage, 56, p. 35-57.

Grosjean, F. (2000) Le droit de l'enfant sourd à grandir bilingue [en ligne], Revue Internationale Surdités, décembre 2000, 3, p. 90-93. Disponible sur : http://www.francoisgrosjean.ch/ the_right_fr.html (consulté le 30 juillet 2018).

Hamm, M. (2010) L'apprentissage de la lecture et de l'écriture chez les personnes sourdes et malentendantes, Thèse de doctorat en Sciences de l'Éducation, Université de Strasbourg.

Hélot, C. \& J. Erfurt (2016) L'éducation bilingue en France. Politiques, linguistiques, modèles et pratiques, Lambert-Lucas.

Hugounenq, H. (2003) L'enseignement de la Langue des Signes aux entendants. Son histoire, ses enjeux au niveau des représentations collectives, ses conséquences concrètes sur la vie des Sourds, Mémoire de DEA en Anthropologie sociale et Ethnologie, EHESS, Paris, 141 p.

Kahne, B-P. (2013) Deaf Tips : Douze leçons du monde des Sourds pour améliorer sa communication personnelle, sociale et professionnelle.

Kerbourc'h, S. (2006) Le Réveil Sourd. D'hier à Aujourd'hui (1971-2006) : de l'action collective d'un mouvement socioculturel pour la réhabilitation de la Langue-des-Signes-Française à la construction d'une identité collective pour la participation sociale des sourds, Thèse de doctorat en Sociologie, EHESS, Paris.

Leroy, É. (2010) Didactique de la langue des signes, langue 1, dans les structures d'éducation en langue des signes. Attitudes et stratégies pédagogiques de l'enseignant sourd, Thèse de doctorat en Sciences du langage, Université Paris 8 - Vincennes-Saint-Denis, Paris.

Mato, O. (2017) Le nouveau paradigme de la surdité et les dynamiques professionnelles : Analyse de la place et du rôle des professionnels entendants de la surdité à partir des années 1970, Thèse de doctorat en Sociologie, Université Paris 8, Paris.

Matsuoka, E. (2016) Études supérieures : visibilité et réalité d'une politique du handicap. Le cas de la surdité, NRAS : Handicap, parole de témoin et parole d'expert : vers une co-construction des discours?, 75 , Suresnes.

Meirieu, P. (2009) Faut-il en finir avec la pédagogie ?, Conférence donnée à Toulouse dans le cadre du GREP le 22 novembre 2009 [en ligne], Toulouse: GREP. Disponible sur :

https://www.meirieu.com/ARTICLES/GREP_CONF_pedagogie.pdf (consulté le 30 juillet 2018).

Meynard, A. (2013) Journal de l'AFILS, 85, sep. 2013, p. 34.

Millet, A. (1999) Orthographe et écriture, langage et surdité : systèmes, représentations, variations, Habilitation à Diriger des Recherches, Université Stendhal, Grenoble 3.

Minguy, A. (2009) Le Réveil Sourd en France. Pour une perspective bilingue. Paris: L'Harmattan. 
Mottez, B. (2006) Les sourds existent-ils? Textes réunis et présentés par A. Benvenuto, Paris: L'Harmattan.

Mottez, B. (1981). La surdité dans la vie de tous les jours [en ligne], Cahiers du CTNERHI, Publications CTNERHI, 114 p. Disponible sur : http://classiques.uqac.ca/contemporains/ handicap_et_inadaptation/documents_CTNERHI/CTNERHI_095/CTNERHI_095.pdf (consulté le 30 juillet 2018).

Perini, M. (2011) Analyse des particularités observées dans des productions écrites d'adultes sourds, Les Actes de Lecture, 114, juin 2011, Paris: Association Française pour la Lecture (AFL).

Terrail, J.-P. (2009) De l'oralité : essai sur l'égalité des intelligences, Paris: La Dispute.

\section{Textes réglementaires}

Bulletins officiels du Ministère de l'Éducation Nationale :

$\mathrm{N}^{\circ} 33$ du 4 septembre 2008

$\mathrm{N}^{\circ} 25$ du 24 juin 2010

Circulaires :

Circulaire $\mathrm{n}^{\circ}$ 2010-068 du 28-5-2010. Bulletin officiel $n^{\circ} 25$ du 24 juin 2010.

NOR : MENE1013746C, MEN - DGESCO - B2-2

Circulaire $n^{\circ}$ 2017-011 du 3 février 2017, pour la Mise en œuvre du parcours de formation du jeune sourd. Bulletin officiel $n^{\circ} 8$ du 23 février 2017.

NOR : MENE1701591C, MENESR - DGESCO A1-3

\section{NOTES}

1. First Cuxac (whose first thesis was on the history of sign language in France, followed by a second one dealing with iconicity from a linguisice viewpoint) and Karacostas (whose thesis in medecine was on the National Institute forthe deaf and dumb in Paris 1790-1800). Then Encrevé and Cantin.

2. In France, mailny Mottez. Then Encrevé and Cantin Kerbourc'h, and Mato more recently.

3. Meynard and Courtin (CNRS researcher in Developmental Cognitive Sciences, dead in 2010).

4. Benvenuto.

5. Humphries and Padden in the USA. Bacci, notably, then Delaporte and Schetrit, in France.

6. Mottez, l'un des premiers chercheurs à avoir accompagné le « réveil sourd » a très bien montré l'importance de voir la surdité comme un rapport, impactant tous les protagonistes dans leurs échanges.

7. Ces stratégies sont d'ailleurs présentées, dans les temps de cours de LSF consacrés à la culture sourde, comme des habitudes de vie des sourds. Quelques-unes ont été explicitées par les travaux en psychologie cognitive de Courtin sur l'attention conjointe, entre autres.

8. Pour Mottez (in Benvenuto, 2006), «La surdité est un rapport » et pour Grosjean (2000), il faut défendre « Le droit de l'enfant sourd à grandir bilingue ».

9. Notre propos ici n'est pas de cerner le particularisme culturel sourd (vécu partagé sans se connaître, sentiment d'appartenance à un groupe de pairs, habitudes de vie, stratégies de communication, adaptation à la vie en société, etc.) Toutefois, comme pour toute autre langue, 
chaque fois qu'il est question de "LSF », de "langue des sourds » ou de «langue gestuelle », la culture qui y est associée devra être prise en considération.

10. La France a signé la charte européenne des langues minoritaires en 1999, mais ne l'a toujours pas ratifiée malgré la promesse de campagnes faite par François Hollande en 2012. Le rapport du comité consultatif pour la promotion des langues régionales et de la pluralité linguistique interne a établi en 2013 une liste des langues susceptibles d'être concernées par la charte et La LSF faisait partie des langues "non territoriales". Le texte a été refusé par le Sénat en octobre 2015.

11. La période nommée communément le "réveil sourd", d'abord par les sourds eux-mêmes mais surtout depuis les travaux de Kerbourc'h en 2006 sur le mouvement qui en découle, commence au milieu des années 1970. Certains auteurs prennent comme point de départ le congrès de la FMS à Paris en 1971, mais il est plus courant de prendre en considération le voyage de Mottez et Deck à Washington en 1975 ou le retour d'un autre voyage de 1978 auquel participaient des professionnels et des familles. La définition d'une balise de fin est plus indécise : le « réveil sourd » s'est-il fini avec la création des premières classes bilingues en 1984 ou avec la reconnaissance de la LSF en 2005 ? Peut-être n'est-il pas encore terminé. La question n'est pas tranchée.

12. Il s'agit ici de la méthode verbo-tonale, ainsi qu'un codage complémentaire à la lecture labiale (LPC), ou encore des technologies avancées dans la reconnaissance vocale.

13. International Visual Theater

14. Académie de la Langue des Signes française

15. Sourds Entendants Recherche Action Communication

16. La reconnaissance officielle de la LSF comme "langue à part entière" ne date que de la loi de février 2005 pour l'égalité des droits et des chances, la participation et la citoyenneté des personnes handicapés (art. L312-9-1, loi n²005-102 du 11 février 2005). C'est un sénateur, Nicolas About, qui est à l'origine d'un amendement adopté à l'unanimité par le Sénat le 1er mars 2004, avant de figurer dans la loi du 11 février 2005. En incitant des jeunes à l'apprendre, il souhaitait avant tout éviter que les sourds et les malentendants ne soient "exclus" du reste du monde. Il espérait même susciter des vocations d'interprètes, trop peu nombreux (une centaine à cette époque), et visait l'objectif d'environ 1600 personnes enseignant dans cette langue.

17. Tout d'abord Cuxac (dont la thèse portait sur l'histoire de la langue des signes en France, alors que la thèse d'état de 1996 portait sur l'iconicité d'un point de vue linguistique) et Karacostas (dont la thèse en médecine portait sur L'Institution nationale des sourds-muets de Paris de 1790 à 1800). Puis Encrevé et Cantin.

18. En France, surtout Mottez. Puis Kerbourc'h, et Mato plus récemment.

19. Meynard et Courtin (chercheur au CNRS en Sciences cognitives développementales, décédé en 2010).

20. Benvenuto.

21. Humphries et Padden aux USA. Bacci, par exemple, puis Delaporte et Schetrit, en France.

22. Cette dénomination proposée en 1988 pour la langue des signes en usage chez les sourds français par Mottez (in Benvenuto, 2006, p. 269) est construite sur le même principe que le sigle américain ASL pour American Sign Language.

23. Ce sera chose faite seulement en 2005 et cette reconnaissance officielle s'est vu réaffirmée par la DGLFLF, qui dépend du Ministère de la Culture, en décembre 2012 (texte archivé et consultable :

http://www.dglflf.culture.gouv.fr/lgfrance/lgfrance_presentation.htm).

24. Voir les travaux de Cuxac (2007 et 2010), Courtin \& Limousin (2010), Leroy (2010), Kahne (2013) et d'autres.

25. Se référer aux chiffres récents de la DEPP (2017) ou encore aux données rassemblées depuis plusieurs années par Ebersold (2013).

TIPA. Travaux interdisciplinaires sur la parole et le langage, 34 | 2018 
26. Quand on recueille les témoignages des sourds (enfants ou des adultes anciens élèves), comme Mottez l'avait fait déjà en 1979 pour La surdité dans la vie de tous les jours, il est difficile de cerner ce qui, au delà de la souffrance personnelle, a permis leur réussite scolaire ou professionnelle. Pour autant, si on ne se donne pas les moyens de recueillir la parole des sourds eux-mêmes, il est absolument aberrant de conclure sur ce que permet ou ne permet pas tel mode de communication ou tel autre.

27. Plusieurs auteurs préconisent de ne pas s'appuyer obligatoirement sur la conscience phonologique pour l'accès à l'écrit. Le premier fut probablement Foucambert et les travaux de l'AFL à sa suite, puis Goigoux (dans Les élèves en grande difficulté de lecture et les enseignements adaptés. CNEFEI, Suresnes, 2000) ou Cambien (dans Apprendre à lire à l'enfant sourd, CNEFEI, Suresnes, 2000) qui s'appuie sur Richaudeau, alors que des travaux comme ceux de Dehaene sont de plus en plus controversés. C'est ce que montre Vanbrugghe (thèse en cours à l'université de Nanterre).

28. Dans le cadre de l'Éducation nationale en France, le FLS est présenté comme langue déterminante pour la socialisation de l'apprenant sourd.

29. Burgat (thèse soutenue, à Paris 8 , en 2007). Voir aussi Besnard (sourd), « Comment construire un pont entre LSF et français pour favoriser l'émergence de l'écrit chez l'enfant sourd scolarisé en classe maternelle bilingue?», mémoire de M2 de Université de Paris 8, soutenu le 14 septembre 2017.

30. Minguy, 2009.

31. Au début des années 1980 , le chercheur américain Krashen oppose l'acquisition naturelle d'une langue en contexte immersif à l'apprentissage ayant lieu en milieu scolaire. Cette dichotomie terminologique a été abandonnée au profit de l'étude des apprentissages «implicites» et «explicites» qu'ils soient observés en milieu scolaire ou en situation d'immersion (Coste, D. 1992).

32. Plus d'information, voir Hélot \& Erfurt (2016).

33. Cf. arrêté du 11 juillet 2017 fixant les programmes d'enseignement de la langue des signes française à l'école primaire et au collège, publication au Journal officiel du 17 août 2017.

34. Nous parlons ici de l'intention sémiotique (Cuxac, 2000). Celle-ci est aussi révélée par l'analyse des langues des signes émergentes (LSE) mises en place par des individus sourds vivant intégrés exclusivement en entourage entendant (cf. les travaux de Fusellier-Souza, 2004). Ceci témoigne du fait que des êtres humains, privés d'un accès direct à un modèle linguistique établi, sont capables de construire un système de communication gestuel linguistiquement organisé. Celui-ci, basé sur le canal visuo-gestuel semble satisfaire les fonctions centrales présentes dans le langage humain.

35. Les créations gestuelles nées du processus d'iconicisation de l'expérience deviennent en quelques années des langues des signes émergentes comme l'a décrit Fusellier-Souza (2004).

36. Minguy, 2009.

37. Voir les travaux d'Ebersold (2013).

38. Le choix de termes pris dans le champ de l'« oralité » appliqué aux langues gestuelles est lié au fait qu'elles sont utilisées en face à face et que, jusqu'à l'utilisation de la vidéo, il n'y avait aucune possibilité d'en garder une trace.

39. Minguy, 2009.

40. Un remaniement est intervenu en 2017.

41. Même le Bureau International d'Audio-Phonologie (BIAP) recommande depuis 2003 une exposition précoce aux deux langues pour tous les enfants sourds.

42. Séminaires STIM organisés par le secteur étudiants de la FNSF et Etudiant's 31, qui réunissent étudiants sourds, interprètes, enseignants, linguistes... autour d'une réflexion sur le lexique spécifique en LSF.

TIPA. Travaux interdisciplinaires sur la parole et le langage, 34 | 2018 
43. Pour plus d'information, lire Dalle, 2005 ou consulter http://anpes.free.fr/Creation/ modeleConfEducBil.pdf

44. Le BELC, né en 1959, a pris son nom actuel en 1965, au moment de son rattachement, comme section spécialisée, au Centre international d'études pédagogiques (CIEP). Il est à l'origine de la revue Le français dans le monde en 1961 et d'un stage d'été régulier qui, au fil des années, a évolué en fonction de l'environnement théorique et des besoins de son public. Il est intéressant de noter que, progressivement, les champs disciplinaires abordés se sont étendus.

45. Le CREDIF, organisme français public de recherche scientifique créé en 1959 également, avait pour objet d'effectuer des recherches et des publications sur l'enseignement du français langue étrangère. La demande ministérielle initiale était d'établir une progression lexicale et syntaxique facilitant la diffusion du français à l'étranger, en réponse à une recommandation de l'Unesco de faciliter l'éducation de masse dans les pays en voie de développement. La création des filières universitaires de FLE a amené le ministère à le supprimer en 1996, ses chercheurs étant répartis dans d'autres unités de l'ENS de Saint-Cloud ou partant dans diverses universités.

46. Cependant, il faut signaler quelques enseignants sourds signants, récemment titularisés sur commission.

47. Ajoutons cependant, pour nuancer nos propos, que la récente révision des programmes (Arrêté du 11 juillet 2017 fixant «les programmes d'enseignement de la langue des signes française à l'école primaire et au collège ", JO 17/08/2017) semble avoir clarifié la place et le rôle de la LSF dans ces cursus bilingues. L'impact de ces choix pourra ainsi être étudié dans quelques années au travers d'une didactique de la LS.

48. Par exemple, la Suède, en 2009, a entériné une modification de la loi qui reconnaît les minorités linguistiques sur son territoire. Il y a cinq langues minoritaires nationales, depuis le 1er janvier 2010 : le finnois, le yiddish, le meänkieli, le rom (romani chib) et le sami. Par ailleurs, faire en sorte que les publics sourds et devenus sourds (même tardivement) aient accès à la langue signée suédoise est considéré comme relevant de la responsabilité collective.

49. Circulaire $n^{\circ}$ 2010-068 du 28-5-2010 du BO n²5 du 24 juin 2010 du Ministère de l'Éducation Nationale.

50. Cf. circulaire $\mathrm{n}^{\circ}$ 2017-011 du 3-2-2017, publiée dans le Bulletin officiel de l'Éducation nationale $\mathrm{n}^{\circ} 8 \mathrm{du} 23 / 02 / 2017$.

51. Nous avons ainsi l'intime conviction que la pédagogie des sourds a tout à nous apprendre puisque l'intention sémiotique des locuteurs sourds crée la langue. "Dire en montrant » est ainsi inhérent à la surdité et cette émergence linguistique se reflète dans les interactions pédagogiques naturelles entre sourds, puisque le pédagogue sourd n'hésite pas à partir à la recherche de cette même intention sémiotique chez l'enfant.

52. Minguy, 2009.

53. La naissance d'une association regroupant ces professionnels prouve ce besoin de partager des savoirs et une expérience commune, cf. https://affels.fr/

54. Sur les obstacles rencontrés par les sourds dans leur parcours à l'université, même en ayant les diplômes exigés, lire E. Matsuoka (2016).

55. Les panels d'élèves de la DEPP : source essentielle pour connaître et évaluer le système éducatif, « Les enfants en situation de handicap. Parcours scolaire à l'école et au lycée ", décembre 2017. Enquête portant sur deux cohortes d'élèves en situation de handicap nés en 2001 et 2005.

56. Mottez et Markowicz avaient débuté une recherche qui a donné lieu, en 1979, au rapport Intégration ou Droit à la différence, Les conséquences d'un choix politique sur les structurations et le mode d'existence d'un groupe minoritaire, les sourds, publié en version bilingue (anglais - français) dans un numéro spécial de la revue Langage en décembre 1979, dir. Grosjean \& Lane).

57. Rappelons que le modèle de Cuxac (2000) établit d'une part, un lien entre les langues des signes émergentes et les langues des signes institutionnelles (lien phylogénétique) et, d'autre 
part, un lien entre les structures grammaticales spécifiques et les unités lexicales (lien diachronique). L'étymologie de ces unités découle d'un processus d'iconicisation de l'expérience.

\section{RÉSUMÉS}

Longtemps évincée du système éducatif pour l'enfant sourd, les études sur la langue des signes française (LSF) et les sourds signeurs se sont inscrites dans le champ universitaire au début des années 1980, en France. Puisque cette langue atypique renvoie davantage à l'universalité de la communication et au rapport au monde qu'à la seule surdité, les questions posées par son enseignement et les spécificités du public concerné sont finalement plus transversales qu'il n'y paraît. Dès lors, les recherches sur la LSF pouvaient relever de plusieurs champs des sciences humaines.

Un bref historique de l'intégration à l'université de cette langue longtemps déconsidérée nous permettra d'analyser l'adéquation de sa place dans les sciences du langage et la posture du chercheur dans ce domaine.

Certes, le « réveil sourd » de la fin des années 1970 s'est nourri de la mobilisation des associations nouvellement créées par le milieu sourd, mais c'est l'histoire des recherches sur la LSF qui nous conduira ici à sonder la place qu'elle pourrait avoir. En entrant dans le panel des sciences du langage, une langue visuelle et minorée comme la LSF a puisé de nouvelles forces. De ce fait, une éventuelle didactique de la LSF peut exister en dehors du champ du handicap. La question qui en découle est alors la pertinence de créer une discipline qui lui soit dédiée.

Cela dit, les recherches sur les sourds et la langue visuo-gestuelle qui leur est intrinsèquement liée apportent également une contribution originale mais dynamique hors du champ de la surdité. Finalement, l'émergence d'idées nouvelles et la définition de concepts inédits issues des travaux sur les sourds dans la société, et en particulier sur leur usage d'une langue gestuelle, président à un bouillonnement épistémologique ayant quelque chose de délicat et de jubilatoire à la fois.

This article offers some insights into which branch of study French sign language and its associated didactics should properly belong to. Our basic premise is that the role of any didactics is to observe teaching phenomena and then construct definitions which can describe them. We shall begin by showing how, despite resistance, the language has become an object of study in universities both as a language taught and a teaching language.

Sign language, like the idea of a specifically deaf culture, has had a troubled history in France. Our aim here is not to dwell on the particularities of deaf culture (shared experience, belonging to a peer group, habitus, communication strategies, adapting to life in a hearing society etc.), but we will nevertheless keep in mind that language and culture are inseparable when discussing language teaching.

French sign language or 'language for the deaf' was denied legitimacy for over a century. It was seen for a long time as a very restricted code, no more than a form of mimicry, which could not be counted as a language. Most notable was the general refusal, from 1880 on, to use the visual resources of sign language in teaching deaf pupils. The issues around teaching the deaf were systematically reduced to the technical question of improving oral French. This focus on oral production and lip-reading meant that little attention was given to what was actually taught, or to teacher-pupil interaction. 
The resulting underlying confusion has persisted, as Mottez puts it: 'since deafness was a pathology, their language would also be one' (Mottez, 1979, in Benvenuto, 2006, p. 252). This is why the recognition of FSL as one of the languages of France in its own right in the early 21st century was such a remarkable advance.

It was only in the 1960s and 70s that linguists such as Stokoe and Markowicz (Gallaudet College, Washington DC, USA) began the formal study of sign language gestures. In their wake, a French researcher named Mottez, a sociologist at the Centre des Mouvements sociaux; Centre for social movements began to take an interest in the deaf community, closely followed by Cuxac, a linguist in France, and Grosjean a psycholinguist in Switzerland.

So it was not until the mid-seventies, with the 'deaf awakening', that the question of which language to use when teaching deaf children could be raised or that any other viewpoint than the dominant one for more than a century could be heard: the idea that a genuine pedagogical relationship can be established with people who 'talk differently', who wish to be understood and who want to learn using a language that reflects their visual mode of thinking.

We should thus note that the initial leverage for a different education for deaf pupils encouraging bilingualism came from research by linguists, who were instrumental in developing associative movements like 2LPE (Deux Langues Pour une Education;Two languages for one education).

Finally, after some fifteen years (1975-90) of discoveries, high hopes and disappointments, this language, the reflection of deaf peoples' way of thinking, would be given official recognition in France. During that time, researchers were working alongside deaf people towards the same goals (Geffroy, 2015; Mato, 2017). Thanks to the combined toolkits of historians ${ }^{1}$, sociologists ${ }^{2}$, psychologists $^{3}$, philosophers ${ }^{4}$, anthropologists and ethnologists ${ }^{5}$, an authentically new, more attentive way of looking at FSL users emerged over thirty years.

FSL was initially introduced into French university curricula as a somewhat 'exotic' subject, which actually became increasingly popular. The first national diploma aimed directly at signing deaf people thus appeared in 2004 as a Continuous Education course at Paris 8 University. This innovation led to a great deal of reflection about possible didactics of FSL, which had so far been given little attention, whether by researchers, deaf or hearing teachers. It must be said that the complex and hitherto unknown situation of French deaf community and their language was indeed a challenge to describe: how could one analyse a language in the absence of any linguistic framework; how could one record it, keep some trace of it? How could people who had been ignored and reduced to silence for so many years be questioned about it?

The study of FSL and its speakers poses both a scientific challenge and an interesting epistemological issue in that it requires us to adjust, if not modify, some deceptively obvious concepts. In particular, 'saying by showing' is inherent to deafness (Cuxac, 2000), and this demonstrative dimension automatically finds its way into any pedagogical interaction led by deaf teachers (Leroy 2010), since a feature of the deaf experience is the practical ability to produce meaning by visual and gestural means, using their own specific proprioceptive cues.

Furthermore, the question of representativity seems to us a crucial one for this population. The category 'hearing impaired' does exist, but is generally insufficiently differentiated: the figures do not tell us who speaks sign language or not, nor with what degree of skill, nor anything about educational experience etc. And yet such details are essential if we are to assess without bias not only the academic skills but also the socialisation and self-esteem opportunities that each particular educational arrangement offers to a deaf child.

All this underlines how unfortunate it would be to ignore the vision that a didactics specific to sign language could provide, whilst playing a support role in educational choices based on objective criteria rather than emotional ones. Indeed, the crucial importance of educational choices for deaf pupils and the atypical way FSL is transmitted both argue cogently for the 
existence of its own specific didactics, articulated around several lines of study: acquisitional, cognitive, linguistic, pedagogical...

In conclusion, we wish to qualify our comments slightly, since the questions raised clearly cannot all be properly dealt with by a didactics of sign-language alone. An international, crossdisciplinary approach is required, involving collaboration with Deaf Studies (or Disabilities Studies) programs in other countries, in order to examine together the ways in which the use of a visualgestural language can contribute to language-teaching didactics generally and, more widely, to the didactics of other academic subjects. Given the strong links that exist between deaf languages in each country, bringing their didactics together would be beneficial for a more distanced approach to deafness, while favouring the emergence of new ways of teaching [transl. by Karen Meschia].

\section{INDEX}

Mots-clés : bilinguisme, langue des signes, LSF, surdité, déficience auditive, éducation, épistémologie, deaf studies

Keywords : bilingualism, sign language, FSL, deafness, hearing impairment, education, epistemology, deaf studies

\section{AUTEURS}

\section{VÉRONIQUE GEFFROY}

INS HEA - Université Paris Lumières, Grhapes EA 7287, Paris, France.

veronique.geffroy@ac-versailles.fr

\section{ÉLISE LEROY}

Université Toulouse 2 Jean Jaurès, CeTIM, CNRS, Laboratoire CLLE, UMR 5263, Toulouse, France. elise.leroy@univ-tlse2.fr 